\title{
Synthesis of Eugenol-Fluorinated Triazole Derivatives and Evaluation of Their Fungicidal Activity
}

\author{
Ângela M. A. Lima, ${ }^{a}$ Wenderson T. de Paula, ${ }^{a}$ Iris C. H. L. Leite, ${ }^{\circledR b}{ }^{b}$ Poliana A. R. Gazolla, ${ }^{c}$ \\ Lucas M. de Abreu, ${ }^{\odot b}$ Victor R. Fonseca, ${ }^{d}$ Wanderson Romão, ${ }^{\circledR d}{ }^{d}$ Valdemar Lacerda Jr., ${ }^{\odot d}$ \\ Vagner T. de Queiroz, ${ }^{a}$ Róbson R. Teixeira ${ }^{\circledR *, c}$ and Adilson V. Costa ${ }^{a}$
}

\author{
${ }^{a}$ Departamento de Química e Física, Universidade Federal do Espírito Santo, 29500-000 Alegre-ES, Brazil \\ ${ }^{b}$ Departamento de Fitopatologia, Universidade Federal de Viçosa, 36570-977 Viçosa-MG, Brazil \\ 'Departamento de Química, Universidade Federal de Viçosa, 36570-977 Viçosa-MG, Brazil \\ ${ }^{d}$ Laboratório de Petroleômica e Forense, Departamento de Química, \\ Universidade Federal do Espírito Santo, 29075-910 Vitória-ES, Brazil
}

\begin{abstract}
Eugenol $\left(\mathrm{C}_{10} \mathrm{H}_{12} \mathrm{O}_{2}\right.$, 4-allyl-2-methoxyphenol) is a phenolic natural product that has several biological activities and possibilities of applications. It is herein described the synthesis of eugenol-fluorinated triazole derivatives and evaluation of their fungicidal activity. The reaction of eugenol with epichlorohydrin resulted in the preparation of ( \pm )-2-((4-allyl-2-methoxyphenoxy) methyl)oxirane (1) in $88 \%$ yield. The azidolysis of $\mathbf{1}$ with sodium azide gave the azido-alcohol ( \pm )-1-(4-allyl-2-methoxyphenoxy)-3-azidopropan-2-ol (2) in 94\% yield. The CuAAc reaction between compound $\mathbf{2}$ and different terminal alkynes afforded a series of eleven derivatives (3a-3k) within $48-80 \%$ yield. All the synthesized compounds were characterized by infrared (IR) and nuclear magnetic resonance $\left({ }^{1} \mathrm{H}\right.$ and $\left.{ }^{13} \mathrm{C}\right)$ spectroscopies and high-resolution mass spectrometry. The in vitro inhibitory activity of the compounds on the mycelial growth of a strain of Colletotrichum sp., that causes anthracnose disease on papaya fruits, was evaluated. The best result was observed for compound 1-(4-allyl-2-methoxyphenoxy)-3-(4-(2-fluorophenyl)-1H-1,2,3-triazol-1-yl)propan-2-ol (3d) that showed a mean growth-inhibition zone of $5.10 \mathrm{~mm}$ in a well-diffusion assay, and may serve as a template for additional structural modifications, aiming for more potent fungicidal activity.
\end{abstract}

Keywords: eugenol, 1,2,3-triazole, fluorinated derivatives, fungicidal activity, papaya anthracnose

\section{Introduction}

The papaya tree fruit (Carica papaya L., Caricaceae family) is originated from the Caribbean coast of Central America and is nowadays cultivated in tropical and subtropical regions of more than 60 countries around the world. ${ }^{1,2}$ It is estimated that papaya fruit global production will increase $2.1 \%$ annually, to reach 16.6 million tons in 2029. ${ }^{3}$

Papaya fruit is regarded as an excellent source of vitamin C. It also contains vitamins A and B3, carotene, riboflavin, fibers, thiamin, niacin, and pantothenic acid. The edible part of the fruit is a source of important minerals such as calcium, potassium, zinc, and iron. Several

*e-mail: robsonr.teixeira@ufv.br

Editor handled this article: Brenno A. D. Neto (Associate) pharmacological properties are associated with the fruit, leaves, and seeds such as anti-helminthic, antiprotozoal, and anti-fertility. The fruit can also help in the prevention of cancer due to the presence of bioactive compounds. , $^{2,-6}$ All of these aspects highlight the nutritive and medicinal values of papaya.

As it happens with several other crops, ${ }^{7,8}$ papaya crop is susceptible to fungal diseases, which must be controlled to avoid losses in production. Among these diseases, it stands out anthracnose, caused by several fungal species of the genus Colletotrichum, ${ }^{9}$ the most important postharvest disease of papaya capable of causing large production losses (up to $90 \%$ ). ${ }^{10-14}$

The infection by Colletotrichum occurs in the field, during fruit development. However, the pathogen remains latent until ripening and harvesting of the fruit, when the fungus colonizes the tissues, leading to the development 
of sunken and necrotic symptoms of anthracnose. The symptoms usually appear during the transport and marketing of papaya. ${ }^{14-16}$ The control of anthracnose is typically made with the periodic application of fungicides because of their low cost, facility of application, and effectiveness. According to the Brazilian Ministry of Agriculture, Livestock and Food Supply (MAPA), there are fourty-seven products registered for papaya anthracnose control based on 15 fungicide molecules, formulated as standalone products or in combinations. ${ }^{17}$ Seven products are made of inorganic copper salts. The most common organic fungicide is mancozeb ( $\mathrm{Mn}$ and Zn-complexed dithiocarbamate) a protectant fungicide component of fourteen standalone and three mix products. The triazole fungicide tebuconazole is the second most common active ingredient present in eleven standalone and two mix formulations used for papaya anthracnose control. Other fungicides used for anthracnose control also include triazoles (difenoconazole, prothioconazole, flutriafol), strobilurins (azoxystrobin, pyraclostrobin, picoxystrobin, trifloxystrobin), and other chemical groups (thiabendazole, fludioxonil, fluxapiroxade, imazali), ${ }^{17}$ (Figure 1).

Within this scenario, fungicides belonging to the class of triazoles have being widely used..$^{14,15,18,19}$ Nonetheless, the use of fungicides can result in the development of fungal species resistance. ${ }^{6,20}$ Other concerns regarding the impact of the use of pesticides (including fungicides) on the environment and human health have prompted researchers to search for new active agents that can be applied at concentrations as low as possible, are safe from the environmental point of view, and possess low toxicity to no-target organisms.

In the search for new fungicides, one important strategy involves the use of secondary metabolites (natural products) produced by plants and other organisms. These compounds can be used directly as fungicides or can be chemically modified to afford hybrid compounds. ${ }^{21,22}$ Several pesticides, ${ }^{23}$ including fungicides, ${ }^{24,25}$ have been discovered by applying this strategy.<smiles>S=C(S)NCCNC(=S)NCCNC(=S)S</smiles><smiles>OC(Cn1cncn1)(c1ccc(F)cc1)c1ccccc1F</smiles>

Flutriafol<smiles>CO/C=C(/C(=O)OC)c1ccccc1COc1cccc(C(F)(F)F)n1</smiles>

Pycostrobin<smiles>N#Cc1c[nH]cc1-c1cccc2c1OC(F)(F)O2</smiles>

Fludioxonil
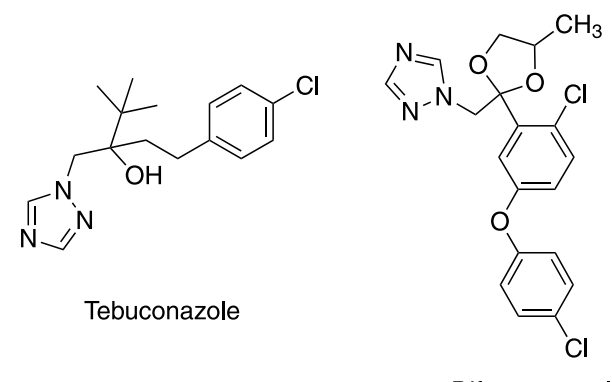

Difenoconazole<smiles>CO/C=C(/C(=O)OC)c1ccccc1Oc1cc(Oc2ccccc2C#N)ncn1</smiles>

Azoxystrobin<smiles>CON=C(C(=O)OC)c1ccccc1CON=C(C)c1cccc(C(F)(F)F)c1</smiles>

Trifloxystrobin

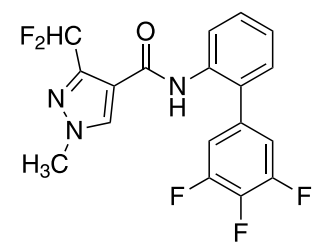

Fluxapiroxade<smiles>COC(=O)N(OC)c1ccccc1COc1ccn(-c2ccc(Cl)cc2)n1</smiles>

Pyraclostrobin<smiles>CCC(Cl)(Cl)C(O)(Cc1ccccc1Cl)Cn1nc[nH]c1=S</smiles><smiles>c1ccc2[nH]c(-c3cscn3)nc2c1</smiles>

Thiabendazole<smiles>C=CCOC(Cn1ccnc1)c1ccc(Cl)cc1Cl</smiles>

Imazali

Figure 1. Structures of organic fungicides effective on the control of Colletotrichum sp. 
Hybrid compounds are obtained by the combination of moieties of different bioactive substances to produce new compounds that may have improved affinity and efficacy as compared to the parent substances. ${ }^{26}$ The 1,2,3-triazoles, one of the most important class of heterocycles, can bind to several biological targets by means of different types of interactions (hydrogen bonds, dipole-dipole, and $\pi$ stacking). ${ }^{27}$ As a consequence, the introduction of a 1,2,3-triazole fragment into the framework of a bioactive compound results in a hybrid with different pharmacological and pharmacokinetic properties and can also afford multitarget derivatives with reduced toxicity and improved efficacy. ${ }^{28}$

Eugenol (4-allyl-2-methoxyphenol) is a natural product found as the major component of the essential oil of cloves (Eugenia caryophyllata). In addition of being a useful building block in organic synthesis, ${ }^{29}$ this phenolic compound and its derivatives possess several biological activities, including insecticide; $;^{30,31}$ antiinflamatory $; 2$ spasmolytic, antisseptic, antipiretic; ${ }^{33}$ antioxidant; ${ }^{34,35}$ neuroprotective; ${ }^{36}$ analgesic, anestesic $;{ }^{37}$ tripanocidal, antimalaric, ${ }^{38}$ antibacterial; ${ }^{39}$ and antifungal. ${ }^{40}$ Our research group has chemically modified eugenol affording compounds with leishmanicidal,${ }^{41}$ antiviral, ${ }^{42}$ and cytotoxic ${ }^{43}$ activities.

We have been involved in the search for new chemical compounds capable of controlling anthracnose. ${ }^{44,45}$ In continuation to our efforts in this field, herein we report the synthesis of new eugenol derivatives bearing 1,2,3-triazolic fragments and the evaluation of their fungicidal activities against a Colletotrichum pathogen that causes anthracnose in papaya.

\section{Experimental}

\section{Generalities}

All reagents and solvents used in this work were purchased, respectively, from Sigma-Aldrich (St. Louis, MO, United States) and Êxodo Científica (Sumaré, São Paulo, Brazil). The thin layer chromatography (TLC) analyses were performed using chromatographic plates of silica gel impregnated on aluminum. After elution, the TLC plates were observed under ultraviolet light $(\lambda=254 \mathrm{~nm})$ and revealed with potassium permanganate solution. Column chromatography separations were performed using silica gel (70-230 mesh, Sigma-Aldrich) as stationary phase. Infrared (IR) spectra were obtained using the attenuated total reflectance (ATR) technique in a Varian 660 instrument (Varian, Palo Alto, CA, USA) equipped with a GladiATr accessory in the $4000-500 \mathrm{~cm}^{-1}$ region. The nuclear magnetic resonance spectra of hydrogen ( ${ }^{1} \mathrm{H}$ NMR, $300 \mathrm{MHz})$ and carbon $\left({ }^{13} \mathrm{C} \mathrm{NMR}, 75 \mathrm{MHz}\right)$ were obtained in a VARIAN MERCURY 300 spectrometer (Varian, Palo Alto, CA, USA), using deuterated chloroform $\left(\mathrm{CDCl}_{3}\right)$ as solvent. The NMR data are presented as follows: chemical shift $(\delta)$ in ppm, multiplicity, the number of hydrogens, and $J$ values in hertz (Hz). Multiplicities are indicated by the following abbreviations: s (singlet), d (doublet), dd (double of doublets), $d_{a p}$ (apparent doublet), ddd (doublet of doublet of doublets), $\mathrm{t}$ (triplet), $\mathrm{td}_{\mathrm{ap}}$ (apparent doublet of triplets), $\mathrm{td}$ (doublet of triplets), q (quartet), quint ${ }_{\mathrm{ap}}$ (apparent quintet), $\mathrm{ddt}_{\mathrm{ap}}$ (apparent doublet of doublets of triplets), and $\mathrm{m}$ (multiplet). The melting temperatures were determined on an MQAPF-302 instrument (Micro Química, Cotia, Brazil) and were uncorrected. The high-resolution mass spectra (HRMS) were obtained on a Solarix 9.4 T instrument (Bruker Daltonics, Bremen, Germany). The samples 3a-3k were dissolved in $1 \mathrm{~mL}$ of acetonitrile and analyzed in an electrospray (ESI) ionization source, operating in the positive mode. The spectrometer was configured to operate in a mass range of $m / z, 150-1500$. The general conditions for analysis by ESI were: nebulizer gas pressure of 1.5 bar, capillary voltage of 4.0-4.4 kV, and capillary transfer temperature of $200^{\circ} \mathrm{C}$.

\section{Synthesis}

\section{Synthesis of 1-(4-allyl-2-methoxyphenoxy)methyl) (1)}

To a $50 \mathrm{~mL}$ round bottom flask containing a magnetic stir bar, it was added eugenol (0.754 g, $4.59 \mathrm{mmol})$, epichlorohydrin ( $4.25 \mathrm{~g}, 45.9 \mathrm{mmol})$, potassium hydroxide $(1.29 \mathrm{~g}, 23.0 \mathrm{mmol})$, and tetrabutylammonium bromide (0.296 g, $0.918 \mathrm{mmol})$. The reaction mixture was kept under magnetic stirring at $0^{\circ} \mathrm{C}$ for $30 \mathrm{~min}$ and for additional $1 \mathrm{~h}$ and $30 \mathrm{~min}$ at room temperature. After the end of the reaction, evidenced by TLC analysis, $20.0 \mathrm{~mL}$ of distilled water were added. Subsequently, the aqueous phase was extracted with dichloromethane $(3 \times 20.0 \mathrm{~mL})$ and the organic extracts were combined. The resulting organic phase was washed with saturated $\mathrm{NaCl}$ solution, dried over anhydrous sodium sulfate, filtered, and concentrated under reduced pressure. The resulting material was purified by silica gel column chromatography using hexane-ethyl acetate $\left(3: 1 \mathrm{v} \mathrm{v}^{-1}\right)$ as eluent. The compound $\mathbf{1}$ was obtained as a colorless oil in $88 \%$ yield $(0.889 \mathrm{~g}, 4.04 \mathrm{mmol})$. The structure of compound $\mathbf{1}$ is supported by the data presented below.

TLC Rf $=0.42$ (hexane-ethyl acetate $3: 1 \mathrm{v} \mathrm{v}^{-1}$ ); IR (ATR) $\mathrm{v} / \mathrm{cm}^{-1} 3060,3000,2921,2837,1635,1591,1510$, 1457, 1419, 1258, 1227, 1139, 1028, 911, 848, 799, 754, 648, 600; ${ }^{1} \mathrm{H}$ NMR $\left(300 \mathrm{MHz}, \mathrm{CDCl}_{3}\right) \delta 2.72(\mathrm{dd}, 1 \mathrm{H}$, $J$ 5.1, $2.7 \mathrm{~Hz}), 2.86-2.89(\mathrm{~m}, 1 \mathrm{H}), 3.32(\mathrm{~d}, 2 \mathrm{H}, J 6.6 \mathrm{~Hz})$, 
3.35-3.39 (m, 1H), $3.85(\mathrm{~s}, 3 \mathrm{H}), 4.02(\mathrm{dd}, 1 \mathrm{H}, J$ 11.4, $5.4 \mathrm{~Hz}), 4.20$ (dd, $1 \mathrm{H}, J 11.4,3.6 \mathrm{~Hz}), 5.03-5.10(\mathrm{~m}, 2 \mathrm{H})$, $5.94\left(\mathrm{ddt}_{\mathrm{ap}}, 1 \mathrm{H}, J 13.5,10.2,6.6 \mathrm{~Hz}\right), 6.69-6.71(\mathrm{~m}, 2 \mathrm{H})$, $6.86(\mathrm{~d}, 1 \mathrm{H}, J 7.8 \mathrm{~Hz}) ;{ }^{13} \mathrm{C}$ NMR $\left(75 \mathrm{MHz}, \mathrm{CDCl}_{3}\right) \delta 39.7$, 44.9, 50.2, 55.8, 70.4, 112.4, 114.4, 115.6, 120.4, 133.8, $137.5,146.3,149.5$.

Synthesis of 1-(4-allyl-2-methoxyphenoxy)-3-azidopropan2-ol (2)

To a $25 \mathrm{~mL}$ round bottom flask, it was added compound 1 ( $0.889 \mathrm{~g}, 4.04 \mathrm{mmol})$, sodium azide $(1.31 \mathrm{~g}$, $20.2 \mathrm{mmol})$, ammonium chloride (0.539 g, $10.1 \mathrm{mmol})$, $4.00 \mathrm{~mL}$ of methanol and $1.00 \mathrm{~mL}$ of distilled water. The reaction mixture remained under magnetic stirring for $3 \mathrm{~h}$ at room temperature. After completion of the reaction as determined by TLC analysis, $20.0 \mathrm{~mL}$ of distilled water was added to the mixture and the aqueous phase was extracted with dichloromethane $(3 \times 20.0 \mathrm{~mL})$. The organic extracts were combined and the resulting organic phase was washed with saturated $\mathrm{NaCl}$ solution, dried over anhydrous sodium sulfate, filtered, and concentrated under reduced pressure. Compound 2 was obtained as a colorless oil in $94 \%$ yield $(1.00 \mathrm{~g}, 3.81 \mathrm{mmol})$. The structure of compound $\mathbf{2}$ is supported by the following data.

TLC Rf $=0.32$ (hexane-ethyl acetate $3: 1 \mathrm{v} \mathrm{v}^{-1}$ ); IR (ATR) $v / \mathrm{cm}^{-1} 3415,3077,3001,2932,2871,2096$, 1636, 1591, 1510, 1455, 1419, 1258, 1227, 1138, 1029, 912, 800, 746, 649; ${ }^{1} \mathrm{H}$ NMR (300 MHz, $\left.\mathrm{CDCl}_{3}\right) \delta 3.33$ $(\mathrm{d}, 2 \mathrm{H}, J 6.6 \mathrm{~Hz}), 3.44(\mathrm{dd}, 1 \mathrm{H}, J 10.5,3.6 \mathrm{~Hz}), 3.50$ (dd, $1 \mathrm{H}, J 12.6,4.8 \mathrm{~Hz}), 3.84(\mathrm{~s}, 3 \mathrm{H}), 3.95-3.98(\mathrm{~m}, 1 \mathrm{H}), 4.03$ $(\mathrm{dd}, 1 \mathrm{H}, J 10.0,4.0 \mathrm{~Hz}), 4.09-4.18(\mathrm{~m}, 1 \mathrm{H}), 5.04-5.11(\mathrm{~m}$, $2 \mathrm{H}), 5.87-6.02(\mathrm{~m}, 1 \mathrm{H}), 6.70-6.72(\mathrm{~m}, 2 \mathrm{H}), 6.85(\mathrm{~d}, 1 \mathrm{H}$, $J 8.4 \mathrm{~Hz}) ;{ }^{13} \mathrm{C} \mathrm{NMR}\left(75 \mathrm{MHz}, \mathrm{CDCl}_{3}\right) \delta 39.83,53.12,55.78$, 69.30, 72.21, 112.43, 115.82, 115.98, 120.81, 134.70, 137.36, 146.11, 149.85 .

Synthesis of eugenol derivatives containing 1,2,3-triazole fragments (3a-3k)

Synthesis of 1-(4-allyl-2-methoxyphenoxy)-3-(4-phenyl1H-1,2,3-triazol-1-yl)propan-2-ol (3a)

A $10 \mathrm{~mL}$ round bottom flask was charged with azide 2 $(0.250 \mathrm{~g}, 0.950 \mathrm{mmol})$, phenylacetylene $(0.0970 \mathrm{~g}$, $0.949 \mathrm{mmol})$, sodium ascorbate $(0.0480 \mathrm{~g}, 0.192 \mathrm{mmol})$, $1.00 \mathrm{~mL}$ of distilled water and $1.00 \mathrm{~mL}$ of dichloromethane. Then, $\mathrm{CuSO}_{4} \cdot 5 \mathrm{H}_{2} \mathrm{O}$ was added. The reaction mixture was kept under stirring for $1 \mathrm{~h}$ at room temperature. After completion of the reaction, as determined by TLC analysis, $20.0 \mathrm{~mL}$ of distilled water was added and the aqueous phase was extracted with dichloromethane $(3 \times 20.0 \mathrm{~mL})$. The organic extracts were combined and the resulting organic phase was washed with saturated ethylenediamine tetraacetic acid (EDTA) solution, dried over anhydrous sodium sulfate, filtered, and concentrated under reduced pressure. The resulting material was purified by silica gel column chromatography using a mixture of hexane-ethyl acetate $\left(3: 2 \mathrm{v} \mathrm{v}^{-1}\right)$ as eluent. Compound 3a was obtained as a white solid in $58 \%$ yield $(0.202 \mathrm{~g}, 0.553 \mathrm{mmol})$. The structure of compound $\mathbf{3 a}$ is in accordance with the following data.

TLC Rf $=0.36$ (hexane-ethyl acetate $3: 2 \mathrm{v} \mathrm{v}^{-1}$ ); mp 93.7-94.2 ${ }^{\circ} \mathrm{C}$; IR (ATR) $v / \mathrm{cm}^{-1} 3321,3137,3065,2935$, 2911, 2872, 1636, 1588, 1510, 1461, 1260, 1226, 1138, 1027, 912, 800, 762, 693, 508, 456; ' $\mathrm{H}$ NMR (300 MHz, $\left.\mathrm{CDCl}_{3}\right) \delta 1.94(\mathrm{~s}, 1 \mathrm{H}), 3.33(\mathrm{~d}, 2 \mathrm{H}, J 6.6 \mathrm{~Hz}), 3.83(\mathrm{~s}, 3 \mathrm{H})$, $3.93(\mathrm{dd}, 1 \mathrm{H}, J 10.2,6.3 \mathrm{~Hz}), 4.06(\mathrm{dd}, 1 \mathrm{H}, J 10.2,4.5 \mathrm{~Hz})$, $4.38-4.47(\mathrm{~m}, 1 \mathrm{H}), 4.55(\mathrm{dd}, 1 \mathrm{H}, J 14.1,6.9 \mathrm{~Hz}), 4.70(\mathrm{dd}$, $1 \mathrm{H}, J 14.1,3.6 \mathrm{~Hz}), 5.01-5.04(\mathrm{~m}, 2 \mathrm{H}), 5.87-6.00(\mathrm{~m}, 1 \mathrm{H})$, 6.68-6.72 (m, 2H), $6.85(\mathrm{~d}, 1 \mathrm{H}, J 8.4 \mathrm{~Hz}), 7.25-7.41(\mathrm{~m}$, $3 \mathrm{H}), 7.75-7.78(\mathrm{~m}, 2 \mathrm{H}), 7.92(\mathrm{~s}, 1 \mathrm{H}) ;{ }^{13} \mathrm{C}$ NMR $(75 \mathrm{MHz}$, $\left.\mathrm{CDCl}_{3}\right) \delta 39.8,52.7,55.7,68.8,71.8,112.4,115.8$, 116.1, 120.9, 121.3, 125.6, 128.0, 128.7, 130.4, 134.9, 137.3, 145.9, 147.5, 149.8; HRMS (ESI) $\mathrm{m} / \mathrm{z}$, calcd. for $\mathrm{C}_{21} \mathrm{H}_{23} \mathrm{~N}_{3} \mathrm{O}_{3}[\mathrm{M}+\mathrm{Na}]^{+}:$388.1631, found: 388.1632 .

The eugenol derivatives $\mathbf{3 b}$-3k were synthesized using a similar methodology to that described for obtaining compound 3a. Information on the reactions involved in the preparation of these compounds and the data supporting their structures are described below.

Synthesis of 1-(4-allyl-2-methoxyphenoxy)-3-(4-(3-fluorophenyl)-1H-1,2,3-triazol-1-yl)propan-2-ol (3b)

White solid, obtained in $63 \%$ yield $(0.191 \mathrm{~g}$, $0.498 \mathrm{mmol})$ from $0.209 \mathrm{~g}(0.793 \mathrm{mmol})$ of the azide, $0.095 \mathrm{~g}(0.793 \mathrm{mmol})$ of the 1-ethynyl-3-fluorobenzene, $0.0630 \mathrm{~g}(0.317 \mathrm{mmol})$ of sodium ascorbate, and $0.039 \mathrm{~g}$ $(0.158 \mathrm{mmol})$ of $\mathrm{CuSO}_{4} \cdot 5 \mathrm{H}_{2} \mathrm{O}$. Reaction time: $1 \mathrm{~h}$. TLC $\mathrm{Rf}=0.33$ (hexane- ethyl acetate $3: 2 \mathrm{v} \mathrm{v}^{-1}$ ); $\mathrm{mp} 96.7-97.4^{\circ} \mathrm{C}$; IR (ATR) $v / \mathrm{cm}^{-1}$ 3163, 3061, 2955, 2931, 2907, 2831, 1618, 1588, 1509, 1464, 1416, 1337, 1256, 1228, 1135, 1023, 914, 863, 785, 739, 662, 597, 525, 456; ' ${ }^{1} \mathrm{H}$ NMR $\left(300 \mathrm{MHz}, \mathrm{CDCl}_{3}\right) \delta 1.94(\mathrm{~s}, 1 \mathrm{H}), 3.33(\mathrm{~d}, 2 \mathrm{H}, J 6.6 \mathrm{~Hz})$, $3.83(\mathrm{~s}, 3 \mathrm{H}), 3.92(\mathrm{dd}, 1 \mathrm{H}, J 10.0,6.0 \mathrm{~Hz}), 4.06(\mathrm{dd}, 1 \mathrm{H}$, $J 10.2,4.5 \mathrm{~Hz}), 4.39-4.46(\mathrm{~m}, 1 \mathrm{H}), 4.56$ (dd, $1 \mathrm{H}, J 14.1$, $6.6 \mathrm{~Hz}), 4.70(\mathrm{dd}, 1 \mathrm{H}, J 14.1,3.6 \mathrm{~Hz}), 5.04-5.11(\mathrm{~m}$, $1 \mathrm{H}), 5.86-6.00(\mathrm{~m}, 1 \mathrm{H}), 6.69-6.72(\mathrm{~m}, 2 \mathrm{H}), 6.85(\mathrm{~d}, 1 \mathrm{H}$, $J 8.4 \mathrm{~Hz}), 6.96-7.03(\mathrm{~m}, 1 \mathrm{H}), 7.35\left(\mathrm{td}_{\mathrm{ap}}, 1 \mathrm{H}, J 6.0,2.1 \mathrm{~Hz}\right)$, 7.46-7.55 (m, 2H), $7.95(\mathrm{~s}, 1 \mathrm{H}) ;{ }^{13} \mathrm{C}$ NMR $(75 \mathrm{MHz}$, $\left.\mathrm{CDCl}_{3}\right) \delta 39.8,52.7,55.7,68.8,71.8,112.4,112.6(\mathrm{~d}$, $J 22.8 \mathrm{~Hz}), 114.8(\mathrm{~d}, J 21.1 \mathrm{~Hz}), 115.8,116.1,120.9$, $121.2(\mathrm{~d}, J 8.3 \mathrm{~Hz}), 121.7,130.4(\mathrm{~d}, J 8.3 \mathrm{~Hz}), 132.6(\mathrm{~d}$, $J$ 8.4 Hz), 134.9, 137.2, 145.8, $146.5(\mathrm{~d}, J 2.8 \mathrm{~Hz}), 149.7$, 
163.1; HRMS (ESI) $m / z$, calcd. for $\mathrm{C}_{21} \mathrm{H}_{22} \mathrm{FN}_{3} \mathrm{O}_{3}[\mathrm{M}+\mathrm{Na}]^{+}$: 406.15374, found: 406.15388 .

Synthesis of 1-(4-allyl-2-methoxyphenoxy)-3-(4-(4-fluorophenyl)-1H-1,2,3-triazol-1-yl)propan-2-ol (3c)

White solid, obtained in $62 \%$ yield $(0.186 \mathrm{~g}$, $0.485 \mathrm{mmol})$ from $0.204 \mathrm{~g}(0.774 \mathrm{mmol})$ of the azide, $0.093 \mathrm{~g}(0.774 \mathrm{mmol})$ of the 1-ethynyl-4-fluorobenzene, $0.0610 \mathrm{~g}(0.309 \mathrm{mmol})$ of sodium ascorbate, and $0.0380 \mathrm{~g}(0.154 \mathrm{mmol})$ of $\mathrm{CuSO}_{4} \cdot 5 \mathrm{H}_{2} \mathrm{O}$. Reaction time: $1 \mathrm{~h}$; TLC Rf $=0.61$ (hexane- ethyl acetate $3: 2 \mathrm{v} \mathrm{v}^{-1}$ ); mp 99.6-100.6 ${ }^{\circ} \mathrm{C}$; IR (ATR) $v / \mathrm{cm}^{-1} 3410,3087,2961,2939$, 2864, 1638, 1605, 1590, 1558, 1509, 1493, 1458, 1416, $1280,1216,1135,1029,917,808,657,594,525 ;{ }^{1} \mathrm{H}$ NMR (300 MHz, $\left.\mathrm{CDCl}_{3}\right) \delta 1.96(\mathrm{~s}, 1 \mathrm{H}), 3.33(\mathrm{~d}, 2 \mathrm{H}, J 6.6 \mathrm{~Hz}$ ), $3.83(\mathrm{~s}, 3 \mathrm{H}), 3.93(\mathrm{dd}, 1 \mathrm{H}, J 10.2,6.3 \mathrm{~Hz}), 4.07(\mathrm{dd}, 1 \mathrm{H}$, $J$ 10.0, $4.2 \mathrm{~Hz}), 4.43(\mathrm{~s}, 1 \mathrm{H}), 4.55$ (dd, $1 \mathrm{H}, J 14.1,6.8 \mathrm{~Hz}$ ), 4.70 (dd, 1H, J 14.1, 3.6 Hz), 5.04-5.09 (m, 2H), 5.86$6.00(\mathrm{~m}, 1 \mathrm{H}), 6.69-6.72(\mathrm{~m}, 2 \mathrm{H}), 6.85(\mathrm{~d}, 1 \mathrm{H}, J 8.7 \mathrm{~Hz})$, $7.08(\mathrm{t}, 2 \mathrm{H}, J 8.7 \mathrm{~Hz}), 7.69-7.74(\mathrm{~m}, 2 \mathrm{H}), 7.89(\mathrm{~s}, 1 \mathrm{H})$; ${ }^{13} \mathrm{C} \mathrm{NMR}\left(75 \mathrm{MHz}, \mathrm{CDCl}_{3}\right) \delta 39.8,52.7,55.7,68.8,71.8$, $112.4,115.8(\mathrm{~d}, J 21.5 \mathrm{~Hz}), 116.2,120.9,121.1,126.7$ (d, $J 3.2 \mathrm{~Hz}), 127.3$ (d, $J$ 8.1 Hz), 134.9, 137.2, 145.8, 146.7, $149.8,162.6$ (d, $J 245.7 \mathrm{~Hz}$ ); HRMS (ESI) $\mathrm{m} / z$, calcd. for $\mathrm{C}_{21} \mathrm{H}_{22} \mathrm{FN}_{3} \mathrm{O}_{3}[\mathrm{M}+\mathrm{Na}]^{+}:$406.15374, found: 406.15362 .

Synthesis of 1-(4-allyl-2-methoxyphenoxy)-3-(4-(2-fluorophenyl)-1H-1,2,3-triazol-1-yl)propan-2-ol (3d)

Colorless oil, obtained in $72.5 \%$ yield $(0.235 \mathrm{~g}$, $0.613 \mathrm{mmol})$ from $0.223 \mathrm{~g}(0.847 \mathrm{mmol})$ of the azide, $0.102 \mathrm{~g}(0.849 \mathrm{mmol})$ of the 1-ethynyl-2-fluorobenzene, $0.0670 \mathrm{~g}(0.339 \mathrm{mmol})$ of sodium ascorbate, and $0.042 \mathrm{~g}$ $(0.169 \mathrm{mmol})$ of $\mathrm{CuSO}_{4} \cdot 5 \mathrm{H}_{2} \mathrm{O}$. Reaction time: $1 \mathrm{~h}$; TLC $\mathrm{Rf}=0.44$ (hexane-ethyl acetate $3: 2 \mathrm{v} \mathrm{v}^{-1}$ ); IR (ATR) $v / \mathrm{cm}^{-1}$ 3339, 3154, 3073, 3004, 2916, 2847, 1636, 1588, 1555, 1509, 1485, 1461, 1419, 1259, 1219, 1138, 1071, 1026, $911,815,755,667,516,468 ;{ }^{1} \mathrm{H} \mathrm{NMR}\left(300 \mathrm{MHz}, \mathrm{CDCl}_{3}\right)$ $\delta 1.85(\mathrm{~s}, 1 \mathrm{H}), 3.32(\mathrm{~d}, 2 \mathrm{H}, J 6.6 \mathrm{~Hz}), 3.84(\mathrm{~s}, 3 \mathrm{H}), 3.94(\mathrm{dd}$, $1 \mathrm{H}, J 10.0,6.0 \mathrm{~Hz}), 4.06(\mathrm{dd}, 1 \mathrm{H}, J 10.2,4.5 \mathrm{~Hz}), 4.40-4.48$ $(\mathrm{m}, 1 \mathrm{H}), 4.58(\mathrm{dd}, 1 \mathrm{H}, J 14.1,6.6 \mathrm{~Hz}), 4.71(\mathrm{dd}, 1 \mathrm{H}, J 14.1$, 4.0 Hz), 5.03-5.09 (m, 2H), 5.86-6.00 (m, 1H), 6.68-6.71 $(\mathrm{m}, 2 \mathrm{H}), 6.85(\mathrm{~d}, 1 \mathrm{H}, J 8.1 \mathrm{~Hz}), 7.06-7.13(\mathrm{~m}, 1 \mathrm{H}), 7.21$ $(\mathrm{dd}, 1 \mathrm{H}, J$ 7.2, $1.5 \mathrm{~Hz}), 7.24-7.30(\mathrm{~m}, 1 \mathrm{H}), 8.10(\mathrm{~d}, 1 \mathrm{H}$, $J 3.9 \mathrm{~Hz}$ ), 8.25 (td, $1 \mathrm{H}, J 7.5,1.8 \mathrm{~Hz}) ;{ }^{13} \mathrm{C}$ NMR $(75 \mathrm{MHz}$, $\left.\mathrm{CDCl}_{3}\right) \delta 38.9,52.6,55.7,68.9,71.8,112.4,115.6(\mathrm{~d}$, $J 21.6 \mathrm{~Hz}), 115.8,116.2,120.8,124.5(\mathrm{~d}, J 3.3 \mathrm{~Hz}), 127.6$ $(\mathrm{d}, J 3.6 \mathrm{~Hz}), 129.2(\mathrm{~d}, J 8.5 \mathrm{~Hz}), 134.9,137.3,141.0(\mathrm{~d}$, $J 2.5 \mathrm{~Hz}), 145.8,149.8,159.2(\mathrm{~d}, J 246.6 \mathrm{~Hz})$; HRMS (ESI) $m / z$, calcd. for $\mathrm{C}_{21} \mathrm{H}_{22} \mathrm{FN}_{3} \mathrm{O}_{3}[\mathrm{M}+\mathrm{Na}]^{+}: 406.15374$, found: 406.15373 .
Synthesis of 1-(4-allyl-2-methoxyphenoxy)-3-(4-(3,4-difluorophenyl)-1H-1,2,3-triazol-1-yl)propan-2-ol (3e)

White solid, obtained in $65 \%$ yield $(0.206 \mathrm{~g}$, $0.513 \mathrm{mmol})$ from $0.209 \mathrm{~g}(0.793 \mathrm{mmol})$ of the azide, $0.109 \mathrm{~g}(0.789 \mathrm{mmol})$ of the 3,4-difluorophenylacetylene, $0.0630 \mathrm{~g}(0.319 \mathrm{mmol})$ of sodium ascorbate, and $0.039 \mathrm{~g}$ $(0.156 \mathrm{mmol})$ of $\mathrm{CuSO}_{4} \cdot 5 \mathrm{H}_{2} \mathrm{O}$. Reaction time: $40 \mathrm{~min}$; TLC $\mathrm{Rf}=0.33$ (hexane-ethyl acetate $3: 2 \mathrm{v} \mathrm{v}^{-1}$ ); $\mathrm{mp} 73.0-73.9^{\circ} \mathrm{C}$; IR (ATR) $v / \mathrm{cm}^{-1} 3290,3139,3112,3078,3006,2942$, 2909, 2834, 1638, 1605, 1563, 1509, 1466, 1433, 1231, 1137, 1122, 1071, 1035, 920, 866, 817, 775, 600, 576; ${ }^{1} \mathrm{H}$ NMR (300 MHz, $\left.\mathrm{CDCl}_{3}\right) \delta 1.95(\mathrm{~s}, 1 \mathrm{H}), 3.33(\mathrm{~d}, 2 \mathrm{H}$, $J 6.6 \mathrm{~Hz}), 3.83$ (s, 3H), 3.90-3.94 (m, 1H), 4.06 (dd, $1 \mathrm{H}$, $J$ 10.2, $4.5 \mathrm{~Hz}$ ), 4.38-4.45 (m, 1H), 4.56 (dd, 1H, $J 14.1$, $6.6 \mathrm{~Hz}), 4.70(\mathrm{dd}, 1 \mathrm{H}, J 14.1,4.0 \mathrm{~Hz}), 5.04-5.10(\mathrm{~m}$, $2 \mathrm{H}), 5.86-5.99(\mathrm{~m}, 1 \mathrm{H}), 6.69-6.72(\mathrm{~m}, 2 \mathrm{H}), 6.85(\mathrm{~d}, 1 \mathrm{H}$, $J 8.1 \mathrm{~Hz}), 7.12-7.21(\mathrm{~m}, 1 \mathrm{H}), 7.45-7.50(\mathrm{~m}, 1 \mathrm{H}), 7.60$ (ddd, $1 \mathrm{H}, J 7.5,2.1 \mathrm{~Hz}), 7.92(\mathrm{~s}, 1 \mathrm{H}) ;{ }^{13} \mathrm{C} \mathrm{NMR}\left(75 \mathrm{MHz}, \mathrm{CDCl}_{3}\right)$ $\delta 39.8,52.7,55.7,68.8,71.8,112.4,114.7(\mathrm{~d}, J 18.5 \mathrm{~Hz})$, $115.9,116.2,117.6(\mathrm{~d}, J 17.3 \mathrm{~Hz}), 120.9,121.5,121.6(\mathrm{dd}$, $J 6.2,3.6 \mathrm{~Hz}$ ), 135.0, 137.2127 .6 (dd, J 6.6, $3.4 \mathrm{~Hz}), 145.8$, 150.2 (dd, J 247.5, 12.6, Hz), 150.6 (dd, J 246.0, $12.4 \mathrm{~Hz}$ ); HRMS (ESI) $m / z$, calcd. for $\mathrm{C}_{21} \mathrm{H}_{21} \mathrm{~F}_{2} \mathrm{~N}_{3} \mathrm{O}_{3}[\mathrm{M}+\mathrm{Na}]^{+}$: 424.14432, found: 424.14424 .

Synthesis of 1-(4-allyl-2-methoxyphenoxy)-3-(4-(2,4-difluorophenyl)-1H-1,2,3-triazol-1-yl)propan-2-ol (3f)

White solid, obtained in $52 \%$ yield $(0.159 \mathrm{~g}$, $0.396 \mathrm{mmol})$ from $0.200 \mathrm{~g}(0.759 \mathrm{mmol})$ of the azide, $0.105 \mathrm{~g}(0.759 \mathrm{mmol})$ of the 1-ethynyl-2,4-difluorobenzene, $0.0600 \mathrm{~g}(0.304 \mathrm{mmol})$ of sodium ascorbate, and $0.0380 \mathrm{~g}$ $(0.152 \mathrm{mmol})$ of $\mathrm{CuSO}_{4} \cdot 5 \mathrm{H}_{2} \mathrm{O}$. Reaction time: $1 \mathrm{~h}$; TLC $\mathrm{Rf}=0.40$ (hexane-ethyl acetate $3: 2 \mathrm{v} / \mathrm{v}$ ); $\mathrm{mp}$ 94.0-95. ${ }^{\circ} \mathrm{C}$; IR (ATR) $v / \mathrm{cm}^{-1} 3342,3154,3071,3001,2919,2850$, $1642,1588,1555,1510,1454,1422,1259,1219,1139$, $1075,1029,915,818,755,673,604,555,513,471$; ${ }^{1} \mathrm{H}$ NMR $\left(300 \mathrm{MHz}, \mathrm{CDCl}_{3}\right) \delta 1.91(\mathrm{~s}, 1 \mathrm{H}), 3.32(\mathrm{~d}, 2 \mathrm{H}$, $J 6.6 \mathrm{~Hz}$ ), 3.83 (s, 3H), 3.94 (dd, 1H, J 10.2, $6.0 \mathrm{~Hz}), 4.07$ $(\mathrm{dd}, 1 \mathrm{H}, J 10.2,4.5 \mathrm{~Hz}), 4.41-4.48(\mathrm{~m}, 1 \mathrm{H}), 4.57(\mathrm{dd}, 1 \mathrm{H}$, $J 14.1,6.6 \mathrm{~Hz}$ ), 4.71 (dd, $1 \mathrm{H}, J 14.1,4.0 \mathrm{~Hz}), 5.04-5.10$ $(\mathrm{m}, 2 \mathrm{H}), 5.86-6.00(\mathrm{~m}, 1 \mathrm{H}), 6.68-6.71(\mathrm{~m}, 2 \mathrm{H}), 6.81-$ $6.89(\mathrm{~m}, 2 \mathrm{H}), 6.93-7.00(\mathrm{~m}, 1 \mathrm{H}), 8.05(\mathrm{~d}, 1 \mathrm{H}, J 4.0 \mathrm{~Hz})$, 8.20 (td, $1 \mathrm{H}, J 8.7,2.4 \mathrm{~Hz}) ;{ }^{13} \mathrm{C} \mathrm{NMR}\left(75 \mathrm{MHz}, \mathrm{CDCl}_{3}\right)$ $\delta 39.8,52.7,55.7,68.9,71.8,104.0(\mathrm{t}, J 25.5 \mathrm{~Hz}), 112.0$ (dd, $J$ 21.1, 3.4 Hz), 112.4, $115.0(\mathrm{dd}, J 13.2,3.7 \mathrm{~Hz})$, $115.8,116.2,120.8,123.9,128.6(\mathrm{dd}, J 9.5,5.1 \mathrm{~Hz})$, 134.9, 137.2, 140.4 (d, J 2.8 Hz), 145.8, 149.8, 159.1 (dd, $J$ 237.4, 7.6 Hz), 162.5 (dd, $J$ 248.9, $12.7 \mathrm{~Hz}$ ); HRMS (ESI) $m / z$, calcd. for $\mathrm{C}_{21} \mathrm{H}_{21} \mathrm{~F}_{2} \mathrm{~N}_{3} \mathrm{O}_{3}[\mathrm{M}+\mathrm{Na}]^{+}: 424.14432$, found: 424.14425 . 
Synthesis of 1-(4-allyl-2-methoxyphenoxy)-3-(4-(3,5-difluorophenyl)-1H-1,2,3-triazol-1-yl)propan-2-ol (3g)

White solid, obtained in $80 \%$ yield $(0.243 \mathrm{~g}$, $0.605 \mathrm{mmol})$ from $0.200 \mathrm{~g}(0.759 \mathrm{mmol})$ of the azide, $0.105 \mathrm{~g}(0.759 \mathrm{mmol})$ of the 1-ethynyl-3,5-difluorobenzene, $0.0600 \mathrm{~g}(0.304 \mathrm{mmol})$ of sodium ascorbate, and $0.0380 \mathrm{~g}$ $(0.152 \mathrm{mmol})$ of $\mathrm{CuSO}_{4} \cdot 5 \mathrm{H}_{2} \mathrm{O}$. Reaction time: $40 \mathrm{~min}$; TLC $\mathrm{Rf}=0.43$ (hexane-ethyl acetate $3: 2 \mathrm{v} \mathrm{v}^{-1}$ ); $\mathrm{mp} 85.0-85.8^{\circ} \mathrm{C}$; IR (ATR) $v / \mathrm{cm}^{-1} 3366,3142,3082,3007,2934,2874$, 1630, 1593, 1510, 1467, 1428, 1364, 1260, 1227, 1117, 1030, 984, 917, 844, 742, 661, 507; ${ }^{1} \mathrm{H}$ NMR $(300 \mathrm{MHz}$, $\left.\mathrm{CDCl}_{3}\right) \delta 1.90(\mathrm{~s}, 1 \mathrm{H}), 3.33(\mathrm{~d}, 2 \mathrm{H}, J 6.6 \mathrm{~Hz}), 3.83(\mathrm{~s}, 3 \mathrm{H})$, 3.88-3-94 (m, 1H), 4.07 (dd, 1H, J 10.0, 4.2 Hz), 4.36-4.44 (m, H-8), 4.56 (dd, $1 \mathrm{H}, J 13.8,6.6 \mathrm{~Hz}$ ), 4.70 (dd, $1 \mathrm{H}, J 14.1$, $4.0 \mathrm{~Hz}), 5.04-5.11(\mathrm{~m}, 2 \mathrm{H}), 5.86-6.00(\mathrm{~m}, 1 \mathrm{H}), 6.69-6.72$ $(\mathrm{m}, 2 \mathrm{H}), 6.76(\mathrm{dt}, 1 \mathrm{H}, J 9.0,2.1), 6.84(\mathrm{~d}, 1 \mathrm{H}, J 8.4 \mathrm{~Hz})$, 7.27-7.34 (m, 2H), $7.97(\mathrm{~s}, 1 \mathrm{H}) ;{ }^{13} \mathrm{C} \mathrm{NMR}\left(75 \mathrm{MHz}, \mathrm{CDCl}_{3}\right)$ $\delta 39.8,52.7,55.7,68.8,71.9,103.3(\mathrm{t}, J 25.2 \mathrm{~Hz}), 108.4(\mathrm{dd}$, $J 18.0,8.6 \mathrm{~Hz}), 112.4,115.9,116.2,120.9,122.1,133.6(\mathrm{t}$, $J 10.5 \mathrm{~Hz}), 135.1,137.2,145.6(\mathrm{t}, J 3.0 \mathrm{~Hz}), 145.8,149.7$, 163.3 (dd, $J$ 246.5, $13.0 \mathrm{~Hz}$ ); HRMS (ESI) $\mathrm{m} / z$, calcd. for $\mathrm{C}_{21} \mathrm{H}_{21} \mathrm{~F}_{2} \mathrm{~N}_{3} \mathrm{O}_{3}[\mathrm{M}+\mathrm{Na}]^{+}:$424.14432, found: 424.14425.

Synthesis of 1-(4-allyl-2-methoxyphenoxy)-3-(4-(4-(trifluoromethyl)phenyl)-1H-1,2,3-triazol-1-yl)propan-2-ol (3h)

White solid, obtained in $50.2 \%$ yield $(0.215 \mathrm{~g}$, $0.496 \mathrm{mmol})$ from $0.260 \mathrm{~g}(0.987 \mathrm{mmol})$ of the azide, $0.168 \mathrm{~g}(0.987 \mathrm{mmol})$ of the 1-ethynyl-4-(trifluoromethyl) benzene, $0.0780 \mathrm{~g}(0.394 \mathrm{mmol})$ of sodium ascorbate, and $0.0490 \mathrm{~g}(0.197 \mathrm{mmol})$ of $\mathrm{CuSO}_{4} \cdot 5 \mathrm{H}_{2} \mathrm{O}$. Reaction time: $1 \mathrm{~h}$; TLC: $\mathrm{Rf}=0.39$ (hexane-ethyl acetate $3: 2 \mathrm{v} \mathrm{v}^{-1}$ ); mp 105.2-106. ${ }^{\circ} \mathrm{C}$; IR (ATR) $v / \mathrm{cm}^{-1} 3417,3136,3079,3046$, 2940, 2883, 2831, 1639, 1618, 1591, 1509, 1458, 1416, 1322, 1229, 1156, 1114, 1062, 1029, 917, 883, 694, 655, 594; ${ }^{1} \mathrm{H}$ NMR (300 MHz, $\left.\mathrm{CDCl}_{3}\right) \delta 1.88(\mathrm{~s}, 1 \mathrm{H}), 3.33(\mathrm{~d}$, $2 \mathrm{H}, J 6.6 \mathrm{~Hz}), 3.83$ (s, 3H), 3.94 (dd, $1 \mathrm{H}, J 10.2,6.3 \mathrm{~Hz}$ ), 4.09 (dd, 1H, J 10.2, $4.2 \mathrm{~Hz}), 4.43-4.46(\mathrm{~m}, 1 \mathrm{H}), 4.57$ (dd, 1H, $J$ 14.1, $6.9 \mathrm{~Hz}), 4.72(\mathrm{dd}, 1 \mathrm{H}, J 14.1,3.6 \mathrm{~Hz})$, 5.05-5.10 (m, 2H), 5.87-6.00 (m, 1H), 6.70-6.73 (m, 2H), $6.85(\mathrm{~d}, 1 \mathrm{H}, J 8.1 \mathrm{~Hz}), 7.63(\mathrm{~d}, 2 \mathrm{H}, J 8.1 \mathrm{~Hz}), 7.86(\mathrm{~d}$, $2 \mathrm{H}, J 6.6 \mathrm{~Hz}), 8.02(\mathrm{~s}, 1 \mathrm{H}) ;{ }^{13} \mathrm{C} \mathrm{NMR}\left(75 \mathrm{MHz}, \mathrm{CDCl}_{3}\right)$ $\delta 39.8,52.8,55.7,68.8,71.9,112.4,115.9,116.2,122.1$, 124.0 (q, $J 268.0 \mathrm{~Hz}), 125.6,125.8$ (q, $J 3.75 \mathrm{~Hz}), 129.9$ (q, $J 32.3 \mathrm{~Hz}), 133.8(\mathrm{~d}, J 1.2 \mathrm{~Hz}), 135.0,137.2,145.8$, 146.1, 149.8; HRMS (ESI) $m / z$, calcd. for $\mathrm{C}_{22} \mathrm{H}_{22} \mathrm{~F}_{3} \mathrm{~N}_{3} \mathrm{O}_{3}$ $[\mathrm{M}+\mathrm{Na}]^{+}:$456.15055, found: 456.15049 .

Synthesis of 1-(4-allyl-2-methoxyphenoxy)-3-(4-(3-(trifluoromethyl)phenyl)-1H-1,2,3-triazol-1-yl)propan-2-ol (3i)

White solid, obtained in $58 \%$ yield $(0.181 \mathrm{~g}$, $0.418 \mathrm{mmol})$ from $0.190 \mathrm{~g}(0.721 \mathrm{mmol})$ of the azide,
$0.123 \mathrm{~g}(0.721 \mathrm{mmol})$ of the 1-ethynyl-3-(trifluoromethyl) benzene, $0.0570 \mathrm{~g}(0.288 \mathrm{mmol})$ of sodium ascorbate, and $0.0360 \mathrm{~g}(0.144 \mathrm{mmol})$ of $\mathrm{CuSO}_{4} \cdot 5 \mathrm{H}_{2} \mathrm{O}$. Reaction time: $40 \mathrm{~min}$; TLC Rf $=0.39$ (hexane-ethyl acetate $3: 2 \mathrm{v} \mathrm{v}^{-1}$ ); $\mathrm{mp}$ 88.8-89.5 ${ }^{\circ} \mathrm{C}$; IR (ATR) $v / \mathrm{cm}^{-1} 3239,3136,3076,3000$, 2937, 2838, 1636, 1591, 1515, 1458, 1419, 1358, 1316, 1228, 1123, 1098, 1071, 1032, 899, 797, 694, 649, 591, 453; ${ }^{1} \mathrm{H}$ NMR (300 MHz, $\left.\mathrm{CDCl}_{3}\right) \delta 1.89(\mathrm{~s}, 1 \mathrm{H}), 3.33(\mathrm{~d}$, $2 \mathrm{H}, J 6.6 \mathrm{~Hz}), 3.83(\mathrm{~s}, 3 \mathrm{H}), 3.92(\mathrm{dd}, 1 \mathrm{H}, J 10.0,6.3 \mathrm{~Hz})$, 4.07 (dd, $1 \mathrm{H}, J 10.2,4.2 \mathrm{~Hz}), 4.39-4.46(\mathrm{~m}, 1 \mathrm{H}), 4.58(\mathrm{dd}$, $1 \mathrm{H}, J 14.1,6.6 \mathrm{~Hz}), 4.72(\mathrm{dd}, 1 \mathrm{H}, J 14.1,3.6 \mathrm{~Hz}), 5.04-$ $5.10(\mathrm{~m}, 2 \mathrm{H}), 5.86-5.99(\mathrm{~m}, 1 \mathrm{H}), 6.70-6.72(\mathrm{~m}, 2 \mathrm{H}), 6.85$ (d, $1 \mathrm{H}, J 8.4 \mathrm{~Hz}), 7.48-7.57(\mathrm{~m}, 2 \mathrm{H}), 7.96-8.02(\mathrm{~m}, 2 \mathrm{H})$, $8.03(\mathrm{~s}, 1 \mathrm{H}) ;{ }^{13} \mathrm{C}$ NMR $\left(75 \mathrm{MHz}, \mathrm{CDCl}_{3}\right) \delta 39.8,52.7$, 55.7, 68.8, 71.9, 112.4, 115.8, 116.3, 120.9, 121.8, 122.4 $(\mathrm{q}, J 3.6 \mathrm{~Hz}), 124.0(\mathrm{q}, J 270.6 \mathrm{~Hz}), 124.7(\mathrm{q}, J 3.6 \mathrm{~Hz})$, 128.7 (q, $J 1.0 \mathrm{~Hz}), 129.3,131.2(\mathrm{q}, J 32.2 \mathrm{~Hz}), 131.3$, 135.0, 137.2, 145.8, 146.2, 149.8; HRMS (ESI) $\mathrm{m} / z$, calcd. for $\mathrm{C}_{22} \mathrm{H}_{22} \mathrm{~F}_{3} \mathrm{~N}_{3} \mathrm{O}_{3}[\mathrm{M}+\mathrm{Na}]^{+}: 456.15055$, found: 456.15049 .

Synthesis of 1-(4-allyl-2-methoxyphenoxy)-3-(4-(2-(trifluoromethyl)phenyl)-1H-1,2,3-triazol-1-yl)propan-2-ol (3j)

Yellow oil, obtained in 59\% yield $(0.202 \mathrm{~g}, 0.466 \mathrm{mmol})$ from $0.209 \mathrm{~g}(0.793 \mathrm{mmol})$ of the azide, $0.135 \mathrm{~g}$ $(0.793 \mathrm{mmol})$ of the 1-ethynyl-2-(trifluoromethyl)benzene, $0.0630 \mathrm{~g}(0.317 \mathrm{mmol})$ of sodium ascorbate, and $0.0390 \mathrm{~g}$ $(0.158 \mathrm{mmol})$ of $\mathrm{CuSO}_{4} \cdot 5 \mathrm{H}_{2} \mathrm{O}$. Reaction time: $2 \mathrm{~h}$; TLC $\mathrm{Rf}=0.36$ (hexane-ethyl acetate $3: 2 \mathrm{v} \mathrm{v}^{-1}$ ); IR (ATR) $\mathrm{v} / \mathrm{cm}^{-1}$ 3375, 3076, 3007, 2937, 2874, 2834, 1636, 1588, 1510, 1464, 1437, 1313, 1260, 1227, 1122, 1107, 1032, 914, 767, 643, 597, 537, 486; ${ }^{1} \mathrm{H}$ NMR (300 MHz, $\left.\mathrm{CDCl}_{3}\right) \delta 1.85$ (s, 1H), $3.33(\mathrm{~d}, 2 \mathrm{H}, J 6.6 \mathrm{~Hz}), 3.83(\mathrm{~s}, 3 \mathrm{H}), 3.91(\mathrm{dd}, 1 \mathrm{H}$, $J$ 10.2, $6.3 \mathrm{~Hz}), 4.05$ (dd, 1H, J 10.2, $4.5 \mathrm{~Hz}), 4.60$ (dd, $1 \mathrm{H}$, $J 14.1,6.6 \mathrm{~Hz}), 4.72(\mathrm{dd}, 1 \mathrm{H}, J 14.1,4.2 \mathrm{~Hz}), 5.03-5.10$ (m, $2 \mathrm{H}), 5.86-6.00(\mathrm{~m}, 1 \mathrm{H}), 6.69-6.72(\mathrm{~m}, 2 \mathrm{H}), 6.85(\mathrm{~d}, 1 \mathrm{H}$, $J 8.4 \mathrm{~Hz}), 7.47(\mathrm{t}, 1 \mathrm{H}, J 7.6 \mathrm{~Hz}), 7.61(\mathrm{t}, 1 \mathrm{H}, J 7.8 \mathrm{~Hz}), 7.74$ (d, $1 \mathrm{H}, J 7.8 \mathrm{~Hz}), 7.92-7.96(\mathrm{~m}, 2 \mathrm{H}) ;{ }^{13} \mathrm{C}$ NMR $(75 \mathrm{MHz}$, $\left.\mathrm{CDCl}_{3}\right) \delta 39.8,52.5,55.7,68.8,71.8,112.3,115.8,116.2$, $120.8,122.2,124.5(\mathrm{q}, J 5.3 \mathrm{~Hz}), 124.0(\mathrm{q}, J 271.8 \mathrm{~Hz})$, 126.0 (q, J $5.7 \mathrm{~Hz}), 127.3$ (q, J $30.1 \mathrm{~Hz}), 128.2,131.6,131.9$ $\left(\mathrm{d}_{\mathrm{ap}}, J 1.0 \mathrm{~Hz}\right), 134.9,137.3,144.1,145.8,149.8$; HRMS (ESI) $m / z$, calcd. for $\mathrm{C}_{22} \mathrm{H}_{22} \mathrm{~F}_{3} \mathrm{~N}_{3} \mathrm{O}_{3}[\mathrm{M}+\mathrm{Na}]^{+}: 456.15 .055$, found: 456.15 .049 .

Synthesis of 1-(4-allyl-2-methoxyphenoxy)-3-(4-(3,5-bis (trifluoromethyl)phenyl)-1H-1,2,3-triazol-1-yl)propan-2-ol (3k)

White solid, obtained in $48.0 \%$ yield $(0.192 \mathrm{~g}$, $0.383 \mathrm{mmol})$ from $0.208 \mathrm{~g}(0.789 \mathrm{mmol})$ of the azide, $0.188 \mathrm{~g}$ $(0.789 \mathrm{mmol})$ of the 1-ethynyl-3,5-bis(trifluoromethyl) benzene, $0.0620 \mathrm{~g}(0.315 \mathrm{mmol})$ of sodium ascorbate, 
and $0.0390 \mathrm{~g}(0.157 \mathrm{mmol})$ of $\mathrm{CuSO}_{4} \cdot 5 \mathrm{H}_{2} \mathrm{O}$. Reaction time: $2 \mathrm{~h}$; TLC Rf $=0.50$ (hexane-ethyl acetate $3: 2 \mathrm{v} \mathrm{v}^{-1}$ ); $\mathrm{mp}$ 100.5-101.1 ${ }^{\circ} \mathrm{C}$; IR (ATR) $v / \mathrm{cm}^{-1}$ 3397, 3140, 3059, 2926, 2853, 1633, 1591, 1512, 1464, 1422, 1388, 1322, $1279,1177,1134,1032,894,806,737,701,680,598$; ${ }^{1} \mathrm{H}$ NMR $\left(300 \mathrm{MHz}, \mathrm{CDCl}_{3}\right) \delta 1.84(\mathrm{~s}, 1 \mathrm{H}), 3.33(\mathrm{~d}, 2 \mathrm{H}$, $J 6.6 \mathrm{~Hz}$ ), 3.84 (s, 3H), 3.90 (dd, 1H, J 10.2, $6.6 \mathrm{~Hz}), 4.08$ (dd, 1H, J 10.0, $4.0 \mathrm{~Hz}), 4.37-4.46(\mathrm{~m}, 1 \mathrm{H}), 4.60(\mathrm{dd}, 1 \mathrm{H}$, $J$ 14.1, 6.6 Hz), 4.74 (dd, 1H, J 14.4, 4.0 Hz), 5.04-5.10 $(\mathrm{m}, 2 \mathrm{H}), 5.86-5.99(\mathrm{~m}, 1 \mathrm{H}), 6.70-6.73(\mathrm{~m}, 2 \mathrm{H}), 6.85(\mathrm{~d}$, $1 \mathrm{H}, J 8.1 \mathrm{~Hz}), 7.81(\mathrm{~s}, 1 \mathrm{H}), 8.15(\mathrm{~s}, 1 \mathrm{H}), 8.25(\mathrm{~s}, 2 \mathrm{H})$; ${ }^{13} \mathrm{C} \mathrm{NMR}\left(75 \mathrm{MHz}, \mathrm{CDCl}_{3}\right) \delta 39.8,52.8,55.7,68.8,71.9$, 112.4, 115.9, 116.3, 120.9, 121.5 (quint $_{\mathrm{ap}}, J 3.5 \mathrm{~Hz}$ ), 122.4, 123.2 (q, J 271.1 Hz), 125.5 (dd, J3.8, 1.0 Hz), 132.7, 132.2 (q, $J 33.2 \mathrm{~Hz}), 135.2,137.2,144.9,145.7,149.7$; HRMS (ESI) $m / z$, calcd. for $\mathrm{C}_{23} \mathrm{H}_{21} \mathrm{~F}_{6} \mathrm{~N}_{3} \mathrm{O}_{3}[\mathrm{M}+\mathrm{Na}]^{+}$: 524.13793, found: 524.13801 .

\section{Evaluation of fungicidal activity}

Stock solutions of eugenol and derivatives $\mathbf{3 a - 3 k}$ in dimethyl sulfoxide (DMSO) $\left(50 \mathrm{mg} \mathrm{mL}^{-1}\right)$ were diluted in a $0.05 \%$ Tween 80 solution to a final concentration of $10 \mathrm{mg} \mathrm{mL}^{-1}$. The same aqueous solution was used as the negative control in the bioassays. The formulated fungicide tebuconazole (Folicur ${ }^{\circledR} 200 \mathrm{EC}, 200 \mathrm{~g} \mathrm{~L}^{-1}$ active ingredient, Bayer) was used as the positive control at the concentration of $10 \mathrm{mg} \mathrm{mL}^{-1}$ of the active ingredient.

One isolate of Colletotrichum sp. (CM01) was obtained from a papaya fruit with typical symptoms of anthracnose. The pathogenicity of the fungus was confirmed by means of artificial inoculation of fruits, following the Koch's postulates. ${ }^{46}$ The fungus was cultivated on potato-dextroseagar medium (PDA) for 7 days at $25^{\circ} \mathrm{C}$.

For the well-diffusion assay, $5 \mathrm{~mm}$ mycelium disks were removed from the edges of the colonies and transferred to one end of $50 \mathrm{~mm}$ Petri dishes containing $5 \mathrm{~mL}$ of PDA. On the opposite sides, 5-mm wells were made in the culture medium and filled with $30 \mu \mathrm{L}$ of each eugenol derivative at $10 \mathrm{mg} \mathrm{mL}^{-1}$ or the control treatments. Plates were incubated at $25^{\circ} \mathrm{C}$ for 6 days. The colony growth-inhibition zone was measured on the sixth day of the experiment using a digital caliper. Three plates were used per sample. The experiment was run twice.

Based on the results of the mycelial growth inhibition with the compounds at $10 \mathrm{mg} \mathrm{mL}^{-1}$, the inhibition of conidia germination and mycelial growth in microtiter plates was used to estimate the minimum inhibitory concentration of eugenol derivatives using a series of doses ranging from 0.5 to $10 \mathrm{mg} \mathrm{mL}^{-1}$. No fungal growth was observed for any treatment at the concentrations of $10 \mathrm{mg} \mathrm{mL}^{-1}$; therefore, this concentration was discarded in the calculation of the $90 \%$ of the maximal effective concentration $\left(\mathrm{EC}_{90}\right)$ values.

The minimum inhibitory concentration (MIC) of compounds was investigated using 96-well microtiter plates. Stock solutions of eugenol and derivatives $\mathbf{3 a - 3 \mathbf { k }}$ in DMSO and the formulated fungicide tebuconazole were serially diluted in a $0.05 \%$ Tween 80 solution to final concentrations of $0,0.5,1.0,2.5$, and $5.0 \mathrm{mg} \mathrm{mL}^{-1}$. Wells in the microtiter plates received $50 \mu \mathrm{L}$ of a suspension of $1 \times 10^{-4}$ conidia $\mathrm{mL}^{-1}$ of the isolate Colletotrichum sp. CM01 in liquid CZAPEK DOX medium, and $50 \mu \mathrm{L}$ of the treatments. The microtiter plates were covered with a plastic wrap, incubated in an orbital shaker at $120 \mathrm{rpm}$ and $25^{\circ} \mathrm{C}$ for $2 \mathrm{~h}$, and transferred to a growth chamber kept at $25^{\circ} \mathrm{C}$ in the dark for four days.

The fungal growth was initially evaluated by measuring the absorbance of each well at $620 \mathrm{~nm}$ after $48 \mathrm{~h}$ using a microplate spectrophotometer. The mixture of liquid CZAPEK DOX and $0.05 \%$ of Tween 80 was used as blank. All treatments were evaluated in triplicates. At the end of the experiment, aliquots of $20 \mu \mathrm{L}$ from each well were inoculated as single points on Petri dishes containing PDA and incubated for four days. The presence or absence of fungal growth at different treatment concentrations was compared to the results of the absorbance test.

\section{Statistical analysis}

Data from the well-diffusion assay were subjected to the Bartlett test to assess the homoscedasticity of the variances, and then adjusted to the linear regression model using the ' $\operatorname{lm}()$ ' function of the lme4 R package. ${ }^{47}$ Means of treatments were grouped by the Scott Knott's cluster test using the scottknott R package..$^{48}$

Absorbance readings from the MIC experiment were modelled using the log-logistic distribution with for parameters, LL.4(), selected using the 'fctlist' function of the drc R package. ${ }^{49}$ The $90 \%$ of the maximal effective concentration $\left(\mathrm{EC}_{90}\right)$ was estimated for each treatment using the function ' $\operatorname{drm}()$ ' of the same package.

\section{Results and Discussion}

Eugenol derivatives containing 1,2,3-triazole fragments 3a-3k were prepared in three steps according to the synthetic route shown in Scheme 1. In the first step, epoxide 1 was prepared in $88 \%$ yield, via bimolecular nucleophilic substitution $\left(\mathrm{S}_{\mathrm{N}} 2\right)$ between eugenol and epichlorohydrin, in the presence of potassium hydroxide and tetrabutylammonium bromide. ${ }^{50}$ Subsequently, epoxide 1 was treated with sodium azide and ammonium 


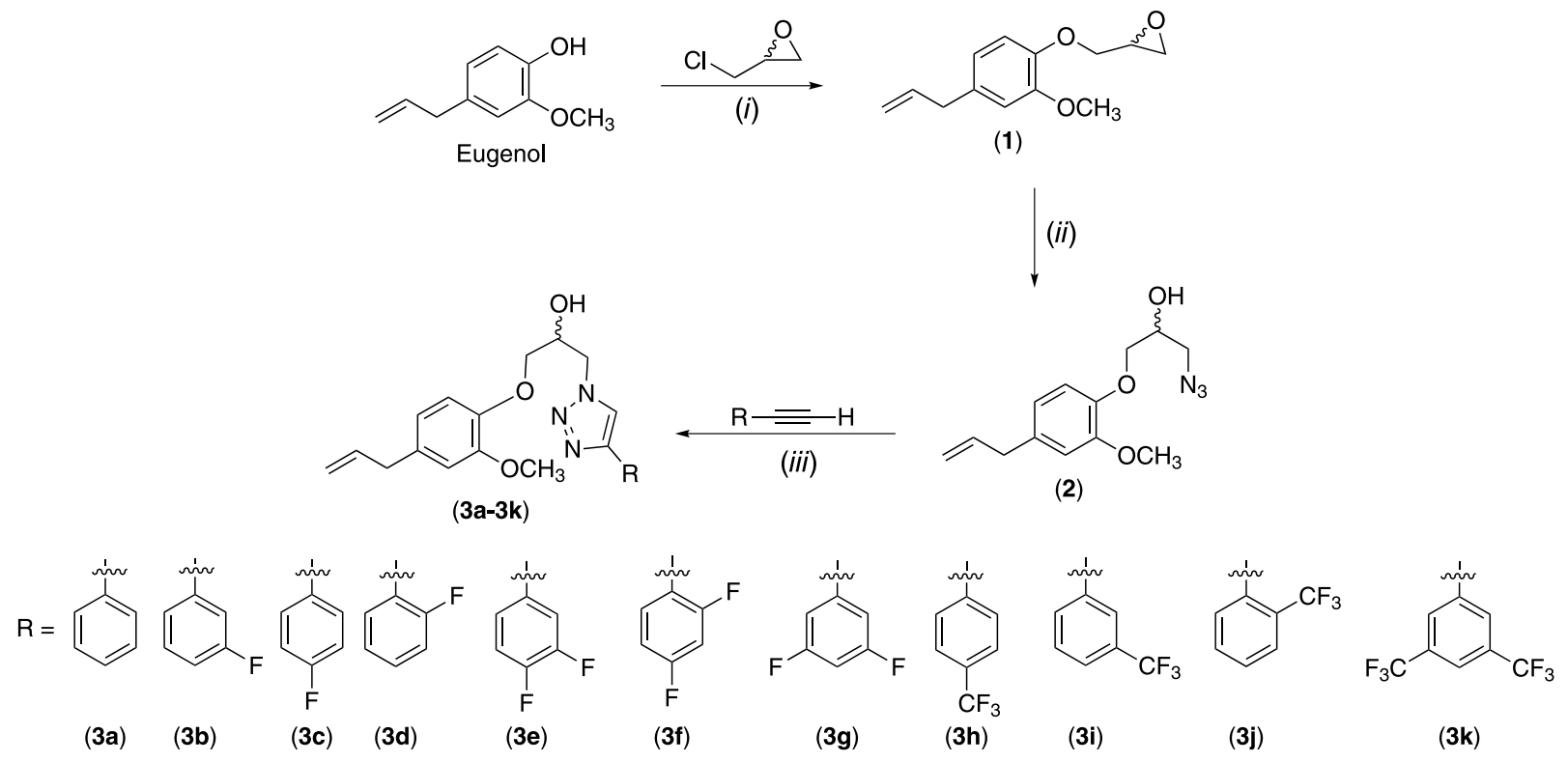

Scheme 1. Synthesis of 1,2,3-triazole compounds derived from eugenol 3a-3k. (i) $\mathrm{KOH}$, $(\mathrm{Bu})_{4} \mathrm{NBr}, 0{ }^{\circ} \mathrm{C} \rightarrow$ r.t., (yield $88 \%$ ); (ii) ammonium chloride, $\mathrm{NaN}_{3}, \mathrm{CH}_{3} \mathrm{OH}, \mathrm{H}_{2} \mathrm{O}, 60{ }^{\circ} \mathrm{C}$ (yield 94\%); (iii) sodium ascorbate, $\mathrm{CuSO}_{4} \cdot 5 \mathrm{H}_{2} \mathrm{O}, \mathrm{CH}_{2} \mathrm{Cl}_{2}, \mathrm{H}_{2} \mathrm{O}$, r.t., (yield 48-80\%).

chloride, resulting in the formation of azide 2 with $94 \%$ yield. ${ }^{51,52}$ In the third and last step, aiming to evaluate the effect of the R-group linked to the triazole portion on the biological activity of the compounds, eleven novel eugenol derivatives 3a-3k were synthesized with yields ranging from $48-80 \%$, via copper(I) catalyzed cycloaddition reactions ${ }^{53,54}$ between azide 2 and different fluorinated aromatic alkynes.

All eugenol triazole derivatives were characterized via infrared (IR) and NMR $\left({ }^{1} \mathrm{H}\right.$ and $\left.{ }^{13} \mathrm{C}\right)$ spectroscopy as well as by high resolution mass spectrometry. In the IR spectra, the band around $1588 \mathrm{~cm}^{-1}$ was attributed to the stretching of the $\mathrm{N}=\mathrm{N}$ bond of the triazole ring. It was also observed the presence of a broad band within $3240-3410 \mathrm{~cm}^{-1}$ range, characteristic of the stretching of $\mathrm{OH}$ group. In the ${ }^{1} \mathrm{H}$ NMR spectra, the signal attributed to the hydrogen present in the triazole ring was observed as a singlet or doublet $(J=4.0 \mathrm{~Hz})$ within the ranges of 7.81-8.03 and 8.05-8.10 ppm, respectively. Signals referring to hydrogen atoms of methylene groups bonded to oxygen (4.55-4.74 ppm) and nitrogen (3.90-4.09 ppm) were generally observed as doublet of doublets. In the ${ }^{13} \mathrm{C}$ NMR spectrum, the triazole ring carbons were observed between approximately 122.0-146.0 ppm. Doublet signals $\left(J_{\mathrm{C}-\mathrm{F}}=244.0-249.0 \mathrm{~Hz}\right)$ relating to carbon couplings with fluorine atoms were also observed at $160.0-164.0 \mathrm{ppm}$. The molecular formulas of the compounds were confirmed by high resolution mass spectrometry analyses.

Once synthesized and characterized, the compounds 3a-3k were subjected to in vitro tests for biological activities. The eugenol derivatives exhibited a moderate inhibitory effect on the mycelial growth of the phytopathogen, when compared with the effect of the fungicide tebuconazole (Figure 2) and eugenol itself (Supplementary Information section, Figure S51). The results were consistent in both repetitions of the experiment (data not shown).

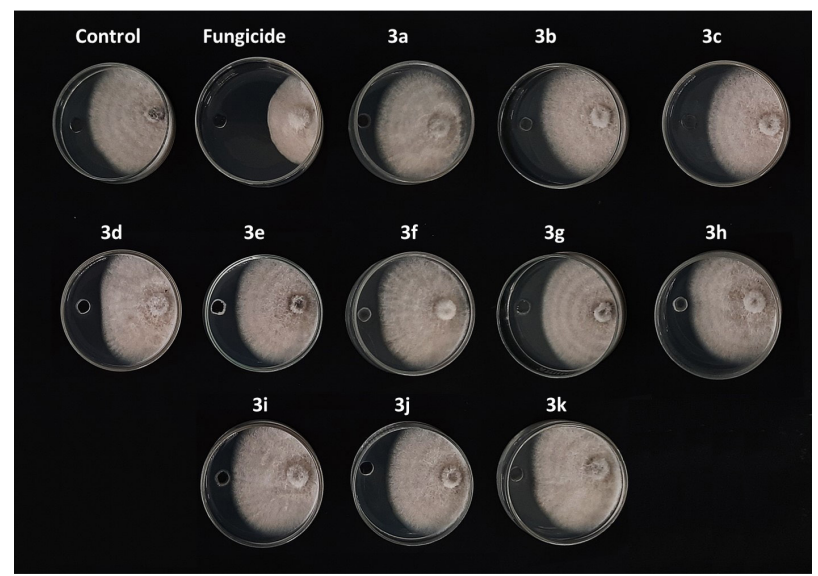

Figure 2. Illustration of the well-diffusion assay against Colletotrichum $\mathrm{sp.}$ Pictures were taken six days after inoculating the phytopathogen on the right side of the Petri dishes.

In the Scott-Knott clustering test, tebuconazole and eugenol formed two singleton cluster with mean growthinhibition zones of 19.0 and $10.7 \mathrm{~mm}$. The third group of treatments contained nine eugenol derivatives. Within this group, the highest mean growth-inhibition zone was $5.1 \mathrm{~mm}$ by derivative $3 \mathbf{d}$ and the lowest $2.3 \mathrm{~mm}$ by derivative $\mathbf{3 b}$ (Figure 3). The mean growth-inhibition zone exhibited by the remaining treatments within the second group varied 
between 3.2 and $3.8 \mathrm{~mm}$. The fourth group of treatments contained the negative control and derivatives $3 \mathbf{i}$ and $\mathbf{3 h}$, both with mean growth-inhibition zones around $1.0 \mathrm{~mm}$.

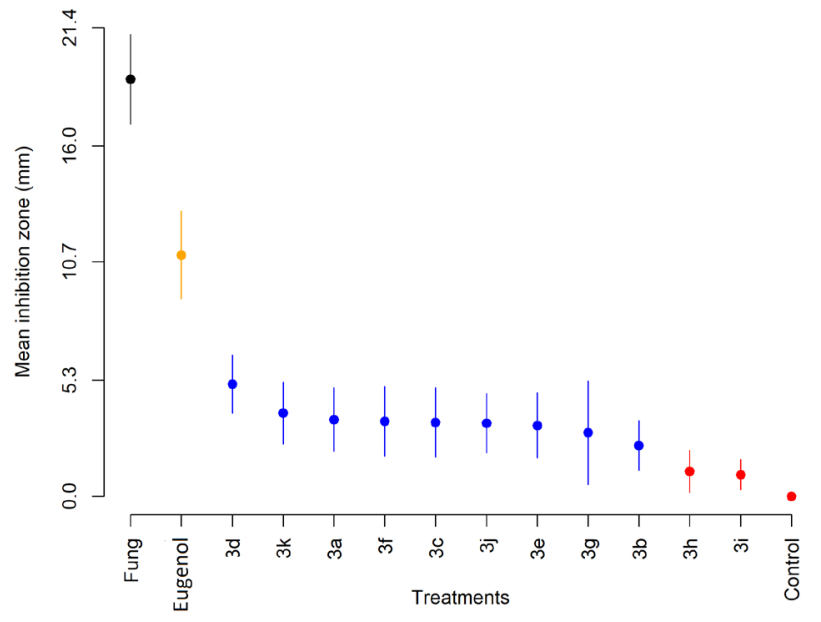

Figure 3. Means and standard deviations of the growth-inhibition zones induced by eugenol derivatives $\mathbf{3 a - 3 k}$ and the fungicide tebuconazole in the well-diffusion assay against Colletotrichum sp. Treatments are grouped by color according to the Scott-Knott test.

The MIC values of all eugenol derivatives fell in the interval between 1.00 and $5.00 \mathrm{mg} \mathrm{mL}^{-1}$ (Table 1). No fungal growth was observed for treatments with eugenol and tebuconazole in the concentrations tested, implying that the MIC for these compounds is smaller than $0.500 \mathrm{mg} \mathrm{mL}^{-1}$.

$\mathrm{EC}_{90}$ estimates of compounds $3 \mathbf{3 f}\left(1.02 \mathrm{mg} \mathrm{mL}^{-1}\right)$ and 3d $\left(1.50 \mathrm{mg} \mathrm{mL}^{-1}\right)$ were the lowest among the eugenol derivatives and corresponded to the MIC interval of $1.00-2.50 \mathrm{mg} \mathrm{mL}^{-1}$ (Table 1). The $\mathrm{EC}_{90}$ of other six derivatives fell between 2.49 and $3.15 \mathrm{mg} \mathrm{mL}^{-1}$ and corresponded to the MIC interval of 2.50 and $5.00 \mathrm{mg} \mathrm{mL}^{-1}$. The $\mathrm{EC}_{90}$ estimates of three eugenol derivatives, based on absorbance readings, fell above the highest dose used; however, samples from the $5.00 \mathrm{mg} \mathrm{mL}^{-1}$ concentration of these treatments failed to grow when inoculated on PDA, suggesting that the $\mathrm{EC}_{90}$ is close, but lower than $5 \mathrm{mg} \mathrm{mL}^{-1}$.

The MIC tests confirmed the bioactivity of $\mathbf{3 d}$ and showed the activity of $\mathbf{3} \mathbf{f}$ in the inhibition of conidial germination and initial fungal growth. The direct effect on the germination of conidia of plant pathogens is desirable in a fungicide molecule, since it prevents the initial infective stages and no disease is observed.

The fungicide activity evaluation revealed that eugenol is more active on Colletotrichum sp. than compounds $\mathbf{3 a - 3 k}$. However, it should be mentioned that the use of eugenol for agrochemical purposes has limitations due to its low stability since it is easily oxidized or decomposed in the presence of oxygen, light, or heat..$^{5,56}$
Table 1. Estimates of the $\mathrm{EC}_{90}$ (absorbance data) and the MIC intervals (no mycelial growth) for the tested compounds

\begin{tabular}{lcc}
\hline Compound & $\mathrm{EC}_{90} /\left(\mathrm{mg} \mathrm{mL}^{-1}\right)$ & $\mathrm{MIC}$ interval $/\left(\mathrm{mg} \mathrm{mL}^{-1}\right)$ \\
\hline 3a & 2.56 & $2.50-5.00$ \\
3b & $>5.00$ & $2.50-5.00$ \\
3c & $>5.00$ & $2.50-5.00$ \\
3d & 1.50 & $1.00-2.50$ \\
3e & $>5.00$ & $2.50-5.00$ \\
3f & 1.02 & $1.00-2.50$ \\
3g & 3.15 & $2.50-5.00$ \\
$3 \mathbf{h}$ & 2.49 & $2.50-5.00$ \\
3i & 2.57 & $2.50-5.00$ \\
3j & 2.54 & $2.50-5.00$ \\
3k & 2.53 & $2.50-5.00$ \\
Eugenol & $<0.500$ & $<0.500$ \\
Tebuconazole & $<0.500$ & $<0.500$ \\
\hline
\end{tabular}

$\mathrm{EC}_{90}: 90 \%$ of the maximal effective concentration; MIC: minimum inhibitory concentration.

The design of fluorine-containing triazole derivatives $\mathbf{3 b} \mathbf{b} \mathbf{3 k}$ was inspired by the fact that several commercial fungicides, namely flutriafol, flusilazole, tetraconazole, epoxiconazole, and fluquinconazole, are triazolic compounds bearing fluorine atoms in their structures. Besides, it is a well-documented the fact that the introduction of fluorine atoms in the design of active ingredients may result in improvement of biological activity. ${ }^{57,58}$ This aspect can be attributed to a combination of properties (such as steric, electronic, and metabolic stability of $\mathrm{C}-\mathrm{F}$ bond) associated with fluorine atom itself. ${ }^{59}$ In the present investigation, compound $\mathbf{3 a}$ was synthesized so that it was possible to assess if the introduction of fluorine groups in the framework of the compounds could afford more potent derivatives. In fact, this was the case since compound 3d, the most active one, presents in its structure a 2-fluorophenyl group.

A comparison of the fungicidal activity of tebuconazole, a 1,2,4 triazole, with the eugenol derivatives containing $1,2,3$ triazole fragments herein investigated cannot be made directly, as the mechanisms of action of these molecules may differ. The 1,2,4 triazoles interact with the enzyme lanosterol $14 \alpha$-demethylase (CYP51), inhibiting ergosterol synthesis in fungi.$^{60}$ The mechanism of interaction between the 1,2,3 triazole fragment and fungal cells is not clear yet. Chloro-containing quinoline derivatives containing 1,2,3 triazole fragments have shown in vitro growth-inhibition zones around $16.9 \mathrm{~mm}$ against the yeast Candida albicans and the filamentous fungus Aspergillus niger, but the fungicidal activity was not solely ascribed to the triazole ring. ${ }^{61}$ 


\section{Conclusions}

In summary, using eugenol as the starting material, a series of fluorinated triazole derivatives were synthesized in three steps. The effect of the compounds was assessed on a Colletotrichum sp., that causes anthracnose in papaya. Derivative 1-(4-allyl-2-methoxyphenoxy)3-(4-(2-fluorophenyl)-1H-1,2,3-triazol-1-yl)propan-2-ol (3d) had the greatest inhibitory effect among the tested substances in the present work and could serve as a template for additional structural modifications, aiming to increase its fungicidal effect.

\section{Supplementary Information}

Supplementary information (IR, NMR and high resolution mass spectra) is available free of charge at http://jbcs.sbq.org.br as PDF file.

\section{Acknowledgments}

We are grateful to Fundação de Amparo à Pesquisa do Estado do Espírito Santo (FAPES), Conselho Nacional de Desenvolvimento Científico e Tecnológico (CNPq), Fundação de Amparo à Pesquisa do Estado de Minas Gerais (FAPEMIG) (APQ-02957-17) for financial support. ICHLL acknowledges the scholarship provided by the Coordenação de Aperfeiçoamento de Pessoal de Nível Superior (CAPES).

\section{Author Contributions}

AMAL was responsible for investigation, data curation, writing original draft, funding acquisition, and writing-review and editing; WTP for investigation and writing-review and editing; ICHLL for investigation, data curation, and writing-review and editing; PARG for data curation, writing original draft, and writing-review and editing; LMA for formal analysis, investigation, validation, writing original draft, and writing-review and editing; VRF, WR, WLJ, VTQ, for formal analysis, data curation, investigation and writing original draft; RRT for conceptualization, formal analysis, investigation, validation, writing original draft, and writing-review and editing; AVC for conceptualization, data curation, formal analysis, funding acquisition, investigation, project administration, resources, validation, writing original draft, and writing-review and editing.

\section{References}

1. Food and Agriculture Organization of the United Nations; Crops-Papaya; https://www.fao.org/in-action/inpho/cropcompendium/en/?ipp=7\&page=3, accessed in August 2021.
2. Saran, P. L.; Choudhary, R. In Achieving Sustainable Cultivation of Tropical Fruits, $1^{\text {st }}$ ed.; Yahia, E. M., ed.; Burleigh Dodds Science Publishing Limited: Cambridge, United Kingdom, 2019, ch. 17.

3. OECD/FAO; OECD-FAO Agricultural Outlook 2021-2030, OECD Publishing: Paris, 2021, available at https://doi. org/10.1787/19428846-en, accessed in January 2022.

4. Alara, O. R.; Abdurahman, N. H.; Alara, J. A.; Adv. Tradit. Med. https://doi.org/10.1007/s13596-020-00481-3.

5. Ikram, E. H. K.; Stanley, R.; Netzel, M.; Fanning, K.; J. Food Compos. Anal. 2015, 41, 201.

6. Rodrigues, J. P.; Coelho, C. C. S.; Soares, A. G.; Otniel FreitasSilva, O.; Biocatal. Agric. Biotechnol. 2021, 36, 102128.

7. Savary, S.; Ficke, A.; Aubertot, J. N.; Hollier, C.; Food Secur. 2012, 4, 519.

8. Almeida, F.; Rodrigues, M. L.; Coelho, C.; Front. Microbiol. 2019, 10, 214.

9. Oliveira, P. D. L.; de Oliveira, K. A. R.; dos Santos Vieira, W. A.; Câmara, M. P. S.; de Souza, E. L.; Int. J. Food Microbiol. 2018, 266, 87.

10. Valenzuela, N. L.; Angel, D. N.; Ortiz, D. T.; Rosas, R. A.; Garcia, C. F.; Santos, M. O.; Biol. Control 2015, 91, 88.

11. Siddiqui, Y.; Ali, A. In Phostarvest Decay: Control Strategies, $1^{\text {st }}$ ed.; Banos, S. B., ed.; Academic Press: Massachusetts, USA, 2014, p. 337.

12. Ribeiro, J. G.; Serra, I. M. R. S.; Araújo, M. U. P.; Summa Phytopathol. 2016, 42, 160.

13. Mangolin, G. S.; Konda, E. T.; Baptista, R. Z.; Nascimento, R. S.; Terao, D.; Braz. J. Anim. Environ. Res. 2019, 2, 1615.

14. Ventura, J. A.; Costa, H.; Tatagiba, J. S. In Manejo das Doenças no Mamoeiro; Martins, D. S.; Costa, A. F. S., eds.; Incaper: Vitória, ES, 2003, p. 229.

15. Fischer, I. H.; de Moraes, M. F.; Palharini, M. C. A.; Cruz, J. C. S.; Firmino, A. N.; ACSA 2017, 13, 130.

16. Vilaplana, R.; Chicaiza, G.; Vaca, C.; Valencia-Chamorro, S.; Crop Prot. 2020, 128, 105007.

17. Ministério da Agricultura, Pecuária e Abastecimento (MAPA); AGROFIT-Sistemas de Agrotóxicos Fitossanitários; https:// agrofit.agricultura.gov.br/agrofit_cons/principal_agrofit_cons, accessed in January 2022.

18. Martins, D. S.; Fornazier, M. J.; Fanton, C. J.; Queiroz, R. B.; Junior, J. S. Z. In Informe Agropecuário: Pragas do Mamoeiro; Epamig: Belo Horizonte, MG, 2016, p. 30.

19. Marzi, M.; Farjam, M.; Kazeminejad, Z.; Shiroudi, A.; Kouhpayeh, A.; Zarenezhad, E.; J. Chem. 2022, 2022, ID 7884316.

20. Tavares, G. M.; Souza, P. E.; Cienc. Agrotecnol. 2005, $29,52$.

21. Lorsbach, B. A.; Sparks, T. C.; Cicchillo, R. M.; Garizi, N. V.; Hahn, D. R.; Meyer, K. G.; Pest Manage. Sci. 2019, 75, 2301.

22. Marrone, P. G.; Pest Manage. Sci. 2019, 75, 2325.

23. Copping, L. G.; Duke, S. O.; Pest Manage. Sci. 2007, 63, 524. 
24. Sauter, H.; Steglich, W.; Anke, T.; Angew. Chem., Int. Ed. 1999, $38,1328$.

25. Loiseleur, O.; Chimia 2017, 77, 810.

26. Xu, Z.; Zhao, S.-J.; Liu, Y.; Eur. J. Med. Chem. 2019, 183, 111700.

27. Dheer, D.; Singh, V.; Shankar, R.; Bioorg. Chem. 2017, 71, 30.

28. Gomtsman, A.; Chem. Heterocycl. Compd. 2012, $48,7$.

29. Kaufman, T. S.; J. Braz. Chem. Soc. 2015, 26, 1055.

30. Maciel, M. V.; Morais, S. M.; Bevilaqua, C. M. L.; Silva, R. A.; Barros, R. S.; Sousa, R. N.; Sousa, L. C.; Brito, E. S.; SouzaNeto, M. A.; Vet. Parasitol. 2010, 167, 1.

31. da Camara, C. A. G.; Doboszewski, B.; de Melo, J. P. R.; Nazarenko, A. Y.; dos Santos, R. B.; Moraes, M. M.; J. Braz. Chem. Soc. 2022, 33, 196.

32. Kim, S. S.; Oh, O. J.; Min, H. Y.; Park, E. J.; Kim, Y.; Park, H. J.; Namhan, Y.; Lee, S. K.; Life Sci. 2003, 73, 337.

33. Feng, J.; Lipton, J. M.; Neuropharmacology 1987, 26, 1775.

34. Guha, S. N.; Priyadarsini, K. I.; Int. J. Chem. Kinet. 1999, 32, 17.

35. Abraham, S. K.; Food Chem. Toxicol. 2001, 39, 493.

36. Kabuto, H.; Tada, M.; Kohno, M.; Biol. Pharm. Bull. 2007, 30, 423.

37. Escobar, R. G.; Rev. Cubana Estomatol. 2002, 39, 139.

38. Teixeira, R. R.; Gazolla, P. A. R.; Silva, S. F.; Aguiar, A. R.; Costa, A. V. In Eugenol: Biosynthesis, Toxicity and Uses; Shelton, M., ed.; Nova Science Pub Inc: New York, 2019, p. 44.

39. Zhang, B.; Eur. J. Med. Chem. 2019, 168, 357.

40. Faria, T. J.; Ferreira, R. S.; Yassumoto, L.; Souza, J. R. P.; Ishikawa, N. K.; Barbosa, A. M.; Braz. Arch. Biol. Technol. 2006, 49, 867.

41. Teixeira, R. R.; Gazolla, P. A. R.; da Silva, A. M.; Borsodi, M. P. G.; Bergmann, B. R.; Ferreira, R. S.; Vaz, B. G.; Vasconcelos, G. A.; Lima, W. P.; Eur. J. Med. Chem. 2018, 146, 274.

42. de Oliveira, A. S.; Gazolla, P. A. R.; Oliveira, A. F. C. S.; Pereira, W. L.; Viol, L. C. S.; Maia, A. F. S.; Santos, E. G.; da Silva, I. E. P.; Mendes, T. A. O.; da Silva, A. M.; Dias, R. S.; da Silva, C. C.; Polêto, M. D.; Teixeira, R. R.; de Paula, S. O.; PLoS One 2019, 14, e0223017.

43. Gazolla, P. A. R.; Teixeira, R. R.; da Silva, A. M.; Vaz, B. G.; Vasconcelos, G. A.; Siqueira, R. P.; Gonçalves, V. H. S.; Pereira, H. S.; Bressan, G. C.; Quim. Nova 2018, 41, 497.
44. Costa, A. V.; de Oliveira, M. V. L.; Pinto, R. T.; Moreira, L. C.; Gomes, E. M. C.; Alves, T. A.; Pinheiro, P. F.; de Queiroz, V. T.; Vieira, L. F. A.; Teixeira, R. R.; Júnior, W. C. J.; Molecules 2017, 22, 1666.

45. Costa, A. V.; Moreira, L. C.; Pinto, R. T.; Alves, T. A.; Schwan, V. V.; de Queiroz, V. T.; Praça-Fontes, M. M.; Teixeira, R. R.; Morais, P. A. B.; de Jesus Jr., W. C.; J. Braz. Chem. Soc. 2020, $31,821$.

46. dos Santos Vieira, W. A.; dos Santos Nunes, A.; Veloso, J. S.; Machado, A. R.; Balbino, V. Q.; da Silva, A. C.; Gomes, A. A. M.; Doyle, V. P.; Câmara, M. P. S.; Australas. Plant Dis. Notes 2020, 15, 2.

47. Bates, D.; Maechler, M.; Bolker, B.; Walker, S.; J. Stat. Software 2015, 67, 1 .

48. Jelihovschi, E. G.; Faria, J. C.; Allaman, I. B.; TEMA 2014, 15, 3.

49. Ritz, C.; Baty, F.; Streibig, J. C.; Gerhard, D.; Plos One 2015, 10, e0146021.

50. Dias, L. C.; Farina, L. S.; Ferreira, M. A. B.; J. Braz. Chem. Soc. 2013, 24, 184.

51. Fringuelli, F.; Piermatti, O.; Pizzo, F.; Vaccaro, L.; J. Org. Chem. 1999, 64, 6094.

52. Amantini, D.; Fringuelli, F.; Piermatti, O.; Tortoioli, S.; Vaccaro, L.; Arkivoc 2002, 2002, 293.

53. Sharpless, K. B.; Finn, M. G.; Kolb, H. C.; Angew. Chem., Int. Ed. 2001, 40, 2004.

54. Meldal, M.; TornØe, C. W.; Chem. Rev. 2008, 108, 2952.

55. Kayaci, F.; Ertas, Y.; Uyar, T.; J. Agric. Food Chem. 2013, 61, 8156.

56. Shimola, K.; Kondo, Y.; Nishida, T.; Hamada, H.; Nakajima, N.; Phytochemistry 2006, 67, 2256.

57. Jeschke, P.; Pest Manage. Sci. 2010, 66, 10.

58. Shah, P.; Westwell, A. D.; J. Enzyme Inhib. Med. Chem. 2007, $22,527$.

59. Jeschke, P.; ChemBioChem 2004, 5, 570.

60. Georgopapadakou, N. H.; Walsh, T. J.; Antimicrob. Agents Chemother. 1996, 40, 279.

61. Kategaonkar, A. H.; Shinde, P. V.; Kategaonkar, A. H.; Pasale, S. K.; Shingate, B. B.; Shingare, M. S.; Eur. J. Med. Chem. 2010, 45, 3142 . 